\title{
RANGE INCLUSION FOR NORMAL DERIVATIONS
}

\author{
by C. K. FONG*
}

In memory of J. P. Williams

(Received 9 May, 1983)

1. Introduction. For a (bounded, linear) operator $A$ in a (complex, infinitedimensional, separable) Hilbert space $\mathscr{H}$, the inner derivation $D_{\mathrm{A}}$, as an operator on $\mathscr{B}(\mathscr{H})$, is defined by $D_{A} X=A X-X A$. Johnson and Williams [4] showed that, when $A$ is a normal operator, range inclusion $D_{B} \mathscr{B}(\mathscr{H}) \subseteq D_{A} \mathscr{B}(\mathscr{H})$ is equivalent to the condition that $B=f(A)$, where $f$ is a Lipschitz function on $\sigma(A)$ such that $t(z, w)(f(z)-f(w)) /(z-w)$ is a trace class kernel on $L^{2}(\mu)$ whenever $t(z, w)$ is such a kernel. (Here $\mu$ is the dominating scalar valued spectral measure of $A$ constructed in multiplicity theory.) This result is deep and its proof is difficult. In the present paper, we establish the following analogous result which is easier to prove: for a normal operator $A$, range inclusion $D_{B} \mathscr{C}_{2}(\mathscr{H}) \subseteq D_{A} \mathscr{C}_{2}(\mathscr{H})$ holds if and only if $B=f(A)$ for some Lipschitz function $f$ on $\sigma(A)$. Here $\mathscr{C}_{2}(\mathscr{H})$ stands for the Hilbert-Schmidt class of operators on $\mathscr{H}$. As by-products of our argument, we generalize some results in [4], [8], [9] concerning the non-existence of a one-sided ideal contained in certain derivation ranges; for example, we show that if $A$ is hyponormal and if the point spectrum $\sigma_{P}\left(A^{*}\right)$ of $A^{*}$ is empty, then $D_{A} \mathscr{B}(\mathscr{H})$ does not contain any nonzero right ideal.

Now let us fix some notation. For $p \geq 1$, we write $\mathscr{C}_{\mathrm{p}}(\mathscr{H})$ for the Schatten $C_{\mathrm{p}}$-class of operators on $\mathscr{H}$ and $\|\cdot\|_{p}$ for its associated $C_{p}$-norm. Let us recall that $\mathscr{B}(\mathscr{H})$ can be regarded as the dual of $\mathscr{C}_{1}(\mathscr{H})$ : each $A \in \mathscr{B}(\mathscr{H})$ determines a bounded linear functional on $\mathscr{C}_{1}(\mathscr{L})$ given by $X \mapsto \operatorname{tr}(A X)$ and vice versa, where $\operatorname{tr}(\cdot)$ stands for the trace of an operator. In the same fashion, $\mathscr{C}_{1}(\mathscr{H})$ can be regarded as the dual of $\mathscr{K}(\mathscr{H})$, the ideal of all compact operators. For $x, y \in \mathscr{H}$, we write $x \otimes y$ for the rank-one operator defined by $(x \otimes y) w=(w, x) y$. The following identities are standard and easy to verify: $(x \otimes y)^{*}=$ $y \otimes x, A(x \otimes y)=x \otimes A y,(x \otimes y) A=A^{*} x \otimes y,\|x \otimes y\|_{p}=\|x\|\|y\|$ and $\operatorname{tr}(x \otimes y)=(y, x)$. For nonzero $y_{0} \in \mathscr{H}$, we write $y_{0} \otimes \mathscr{H}$ for the minimal left ideal $\left\{y_{0} \otimes x: x \in \mathscr{H}\right\}$ and $\mathscr{H} \otimes y_{0}$ for the minimal right ideal $\left\{x \otimes y_{0}: x \in \mathscr{H}\right\}$.

2. Right ideals in a derivation range. The following lemma on range inclusion can be found in [2] and [4]. A short proof is included for the reader's convenience.

LEMMA 2.1. If $\mathscr{X}_{1}, \mathscr{X}_{2}, \mathscr{Y}$ are Banach spaces, if $S: \mathscr{X}_{1} \rightarrow \mathscr{Y}$ and $T: \mathscr{X}_{2} \rightarrow \mathscr{Y}$ are bounded linear maps, and if $S \mathscr{X}_{1} \subset T \mathscr{X}_{2}$, then there exists a positive constant $k$ such that $\left\|S^{\prime} \phi\right\| \leq$ $k\left\|T^{\prime} \phi\right\|$ for all $\phi \in \mathcal{Y}^{\prime}$. (Here $\mathscr{Y}^{\prime}$ stands for the dual of $\mathscr{Y}$ and $S^{\prime}$ stands for the transpose of $S$.)

\footnotetext{
* The author would like to thank the Natural Sciences and Engineering Research Council of Canada for the financial support.
}

Glasgow Math. J. 25 (1984) 255-262 
Proof. Let $\tilde{\mathscr{X}}_{2}$ be the quotient space $\mathscr{X}_{2} /$ ker $T$ and $Q: \mathscr{X}_{2} \rightarrow \mathscr{\mathscr { X }}_{2}$ be the quotient map. Then $T$ induces a map $\tilde{T}: \tilde{\mathscr{X}}_{2} \rightarrow \mathscr{Y}$ such that $\tilde{T} Q=T$. Since $\tilde{T}$ is one-one and $S \mathscr{X}_{1} \subset \tilde{T} \tilde{\mathscr{Z}}_{2}$, there is a unique linear map $A: \mathscr{X}_{1} \rightarrow \tilde{\mathscr{X}}_{2}$ such that $S=\tilde{T} A$. By means of the closed graph theorem, it is easy to check that $A$ is bounded. For $\phi \in \mathscr{Y}^{\prime}$, since $Q^{\prime}: \tilde{\mathscr{X}}_{2}^{\prime} \rightarrow \mathscr{X}_{2}^{\prime}$ is an isometry, we have

$$
\left\|S^{\prime} \phi\right\|=\left\|A^{\prime} \tilde{T}^{\prime} \phi\right\| \leq\left\|A^{\prime}\right\|\left\|\tilde{T}^{\prime} \phi\right\|=\left\|A^{\prime}\right\|\left\|Q^{\prime} \tilde{T}^{\prime} \phi\right\|=\left\|A^{\prime}\right\|\left\|T^{\prime} \phi\right\| .
$$

Therefore we may choose $\|A\|$ to be $k$.

THEOREM 2.2. If $A \in \mathscr{B}(\mathscr{H}), y_{0} \in \mathscr{H}$ and $\mathscr{H} \otimes y_{0} \subseteq D_{\mathrm{A}} \mathscr{B}(\mathscr{H})$, then there exists $k>0$ such that

(1) $\left\|X y_{0}\right\| \leq k\|A X-X A\|_{1}$

for all $X \in \mathscr{C}_{1}(\mathscr{H})$ and, furthermore,

(2) $y_{0} \in \bigcap\left\{(A-\lambda) \mathscr{H}: \lambda \in \sigma_{a p}(A)\right\}$,

where $\sigma_{a p}(A)$ stands for the approximate point spectrum of $A$.

Proof. For convenience, we write $B=A^{*}$. Then, by taking adjoints, we see that the condition $\mathscr{H} \otimes y_{0} \subseteq D_{\mathrm{A}} \mathscr{B}(\mathscr{H})$ is equivalent to $y_{0} \otimes \mathscr{H} \subseteq D_{\mathrm{B}} \mathscr{B}(\mathscr{H})$. Each $X$ in $\mathscr{C}_{1}(\mathscr{H})$ gives rise to a linear functional $\phi_{\mathrm{X}}$ on $\mathscr{B}(\mathscr{H})$ given by $\phi_{X}(Y)=\operatorname{tr}(X Y)$. Hence we may identify $\mathscr{C}_{1}(\mathscr{H})$ with a subspace of $\mathscr{B}(\mathscr{H})^{\prime}$. On this subspace, we have $D_{B}^{\prime} X=X B-B X$, since for $Y \in \mathscr{B}(\mathscr{H})$, we have $\left(D_{B}^{\prime} X\right)(Y)=\operatorname{tr}\left(X\left(D_{B} Y\right)\right)=\operatorname{tr}(X B Y-X Y B)=\operatorname{tr}(X B Y-B X Y)=$ $\operatorname{tr}((X B-B X) Y)$. Define $S: \mathscr{H} \rightarrow \mathscr{B}(\mathscr{H})$ by $S x=y_{0} \otimes x$. Then, for $X \in \mathscr{C}_{1}(\mathscr{H})$ and $x \in \mathscr{H}$, we have

$$
\left(S^{\prime} X\right)(x)=\operatorname{tr}(X(S x))=\operatorname{tr}\left(X\left(y_{0} \otimes x\right)\right)=\operatorname{tr}\left(y_{0} \otimes X x\right)=\left(X x, y_{0}\right)=\left(x, X^{*} y_{0}\right) .
$$

Hence $\left\|S^{\prime} X\right\|=\left\|X^{*} y_{0}\right\|$. By Lemma 2.1 , there is a positive constant $k$ such that $\left\|S^{\prime} X\right\| \leq$ $k\left\|D_{B}^{\prime} X\right\|$ for $X \in \mathscr{C}_{1}(\mathscr{H})$, or

$$
\left\|X^{*} y_{0}\right\| \leq k\left\|A^{*} X-X A^{*}\right\|_{1} .
$$

Replacing $X^{*}$ by $X$, we obtain (1).

Let $\lambda \in \sigma_{a p}(A)$. We have to exhibit $x_{0} \in \mathscr{H}$ such that $y_{0}=(A-\lambda) x_{0}$. Let $\left\{x_{n}\right\}$ be a sequence of unit vectors such that $\left\|(A-\lambda) x_{n}\right\| \rightarrow 0$. We put $X=y \otimes x_{n}$ in (1) to obtain

By letting $n \rightarrow \infty$, we have

$$
\begin{aligned}
\left|\left(z, y_{0}\right)\right| & =\left\|\left(y_{0}, z\right) x_{n}\right\|=\left\|\left(z \otimes x_{n}\right) y_{0}\right\| \\
& \leq k \mid(A-\lambda)\left(z \otimes x_{n}\right)-\left(z \otimes x_{n}\right)(A-\lambda) \|_{1} \\
& =k\left\|z \otimes(A-\lambda) x_{n}-\left((A-\lambda)^{*} z\right) \otimes x_{n}\right\|_{1} \\
& \leq k\|z\|\left\|(A-\lambda) x_{n}\right\|+k\left\|(A-\lambda)^{*} z\right\| .
\end{aligned}
$$

$$
\left|\left(z, y_{0}\right)\right| \leq k\left\|(A-\lambda)^{*} z\right\| \text {. }
$$

The mapping sending $(A-\lambda)^{*} z$ to $\left(z, y_{0}\right)$ is well-defined, linear and bounded. It can be extended to a bounded linear functional on the whole space $\mathscr{H}$. Hence, by the Riesz 
representation theorem, there exists $x_{0} \in \mathscr{H}$ such that $\left((A-\lambda)^{*} z, x_{0}\right)=\left(z, y_{0}\right)$ for all $x \in \mathscr{H}$. Therefore $y_{0}=(A-\lambda) x_{0}$.

COROLlary 2.3 (Johnson and Williams [4, Theorem 2.5]). If $A$ is a normal operator, then $D_{A} \mathscr{B}(\mathscr{H})$ does not contain any nonzero right ideal.

Proof. This result is an immediate consequence of the above theorem and the following lemma.

Lemma 2.4 (Johnson [3], Putnam [5]). If $A$ is a normal operator, $E$ is its spectral measure and $\delta$ is a closed set in $\sigma(A)$, then

in particular

$$
E(\delta) H=\bigcap\{(A-\lambda) \mathscr{H}: \lambda \notin \delta\}
$$

$$
\bigcap\{(A-\lambda) \mathscr{H}: \lambda \in \mathbb{C}\}=\{0\} .
$$

COROLlary 2.5 (Williams [9, Theorem 1]). If $S$ is an isometry, then $D_{S} \mathscr{B}(\mathscr{H})$ does not contain any nonzero right ideal.

Proof. If $S S^{*}=I$, then $S$ is unitary, and this case is covered by Corollary 2.3. Hence we assume that $S S^{*} \neq I$. Therefore the unit circle $T$ is contained in $\sigma_{a p}(S)$. Suppose on the contrary that $\mathscr{H} \otimes y_{0} \subseteq D_{S} \mathscr{B}(\mathscr{H})$ for some $y_{0} \neq 0$. By Theorem 2.2, we have $y_{0} \in$ $\bigcap\{(S-\lambda) \mathscr{H}: \lambda \in \mathbb{T}\}$. Let $U \in \mathscr{B}(\mathscr{K})$ be a unitary extension of $S$. Then we have $y_{0} \in$ $\bigcap\{(U-\lambda) \mathscr{K}: \lambda \in \mathbb{T}\}$, contradictory to Lemma 2.4 .

From the proof of the above corollary we see that if $S$ is a subnormal operator and $\sigma(N) \subseteq \sigma_{a p}(S)$, where $N$ is the minimal normal extension of $S$, then $D_{S} \mathscr{B}(\mathscr{H})$ does not contain any nonzero right ideal.

Lemma 2.4 can be generalized for hyponormal operators (see Clancy [1]): if $A$ is a hyponormal operator, then $\bigcap\{(A-\lambda) \mathscr{H}: \lambda \in \mathbb{C}\}=\{0\}$. From this result and Theorem 2.2 we obtain the following corollary.

COROLlARY 2.6. If $A$ is a hyponormal operator and if $D_{A} \mathscr{B}(\mathscr{H})$ contains a nonzero right ideal, then $\sigma_{a_{p}}(A) \neq \sigma(A)$ and, in particular, $\sigma_{\mathrm{p}}\left(A^{*}\right)$ is nonempty.

COROLlaRY 2.7. If $A \in \mathscr{B}(\mathscr{H})$ and if there exists a sequence $\left\{F_{n}\right\}$ of finite rank operators such that the linear span of their ranges is dense in $\mathscr{H}$ and $A F_{n}^{*}=F_{n}^{*} A$ for all $n$, then $D_{A} \mathscr{B}(\mathscr{H})$ does not contain any nonzero right ideal.

Proof. Suppose that $\mathscr{H} \otimes y_{0} \subseteq D_{A} \mathscr{B}(\mathscr{H})$. Then, by Theorem 2.2 , there exists $k>0$ such that $\left\|X y_{0}\right\| \leq k\|A X-X A\|_{1}$. Hence $F_{n}^{*} y_{0}=0$ for all $n$. In other words, $y_{0}$ is perpendicular to the range of $F_{n}$ for all $n$. Therefore $y_{0}=0$.

Corollary 2.8 (Stampfli [7]). For each $A \in \mathscr{B}(\mathscr{H}), D_{\mathrm{A}} \mathscr{B}(\mathscr{H})$ does not contain any nonzero two-sided ideal.

Proof. Suppose to the contrary that $D_{A} \mathscr{B}(\mathscr{H})$ contains all finite rank operators. Then, for each $y_{0} \in \mathscr{H}, \mathscr{H} \otimes y_{0} \subseteq D_{A} \mathscr{B}(\mathscr{H})$ and hence, by Theorem 2.2, $y_{0} \in(A-\lambda) \mathscr{H}$ for $\lambda \in$ $\sigma_{a p}(A)$. Therefore $A-\lambda$ is surjective for all $\lambda \in \sigma_{a p}(A)$. On the other hand, for each 
$y_{0} \in \mathscr{H}, y_{0} \otimes \mathscr{H} \subseteq D_{\mathrm{A}} \mathscr{B}(\mathscr{H})$ and hence $\mathscr{H} \otimes y_{0} \subseteq D_{\mathrm{A}} * \mathscr{B}(\mathscr{H})$. In the same way, we see that $A^{*}-\mu$ is surjective for $\mu \in \sigma_{a p}\left(A^{*}\right)$. Now let $\lambda_{0}$ be a point at the boundary of $\sigma(A)$. Then $\lambda_{0} \in \sigma_{a p}(A)$ and $\bar{\lambda}_{0} \in \sigma_{a p}\left(A^{*}\right)$. Hence both $A-\lambda_{0}$ and $\left(A-\lambda_{0}\right)^{*}$ are surjective, contradicting the fact that $\lambda_{0} \in \sigma(A)$.

\section{Rank one majorization.}

THEOREM 3.1. If $A, B \in \mathscr{B}(\mathscr{H})$ and if $\left\|D_{\mathrm{B}} X\right\| \leq k\left\|D_{\mathrm{A}} X\right\|_{\mathrm{p}}$ for all rank one operators $X$, then we have

(1) for each $\lambda \in \sigma_{a p}\left(A^{*}\right)^{*}$, there exists $\mu \in \mathbb{C}$ such that, for all $x \in \mathscr{H}$,

$$
\|(B-\mu) x\| \leq k\|(A-\lambda) x\|
$$

(2) there is a Lipschitz function $f$ on $\sigma_{a p}(A) \cap \sigma_{a p}\left(A^{*}\right)^{*}$ such that, for all $x \in \mathscr{H}$ and $\lambda \in \sigma_{a p}(A) \cap \sigma_{a p}\left(A^{*}\right)^{*}$, we have

$$
\begin{aligned}
\|(B-f(\lambda)) x\| & \leq k\|(A-\lambda) x\|, \\
\left\|(B-f(\lambda))^{*} x\right\| & \leq k\left\|(A-\lambda)^{*} x\right\| .
\end{aligned}
$$

Proof. Assume that $\bar{\lambda} \in \sigma_{a p}\left(A^{*}\right)$. Then there exists a sequence $\left\{x_{n}\right\}$ of unit vectors in $\mathscr{H}$ such that $\left\|(A-\lambda)^{*} x_{n}\right\|$ tends to zero. Now

$$
\left\|D_{\mathrm{A}}\left(x_{n} \otimes y\right)\right\|_{p}=\left\|x_{n} \otimes((A-\lambda) y)-\left((A-\lambda)^{*} x_{n}\right) \otimes y\right\|_{p},
$$

which tends to $\|(A-\lambda) y\|$. On the other hand,

$$
\begin{aligned}
\left\|D_{B}\left(x_{n} \otimes y\right)\right\| & \geq\left\|\left(D_{B}\left(x_{n} \otimes y\right)\right) x_{n}\right\| \\
& =\left\|\left(x_{n} \otimes B y\right) x_{n}-\left(B^{*} x_{n} \otimes y\right) x_{n}\right\|=\left\|B y-\left(B x_{n}, x_{n}\right) y\right\| .
\end{aligned}
$$

Therefore, if $\mu$ is a cluster point of the bounded sequence $\left\{\left(B x_{n}, x_{n}\right)\right\}$, then

(a) $\|(B-\mu) y\| \leq k\|(A-\lambda) y\|$.

Thus we have proved (1).

Note that the relation $\left\|D_{\mathrm{B}} X\right\| \leq k\left\|D_{\mathrm{A}} X\right\|_{\mathrm{p}}$ is equivalent to $\left\|D_{\mathrm{B}^{*}} Y\right\| \leq k\left\|D_{\mathrm{A}^{*}} Y\right\|_{\mathrm{p}}$, where $Y=X^{*}$. Hence we can apply (1) for $A^{*}, B^{*}$ to conclude that, for each $\lambda \in \sigma_{a p}(A)$, there exists $\mu^{\prime} \in \mathbb{C}$ such that, for $x \in \mathscr{H}$,

(b) $\left\|\left(B-\mu^{\prime}\right)^{*} x\right\| \leq k\left\|(A-\lambda)^{*} x\right\|$.

Now we suppose $\lambda \in \sigma_{a p}(A) \cap \sigma_{a p}\left(A^{*}\right)^{*}$. Then there exists a sequence $\left\{x_{n}\right\}$ of unit vectors such that $\left\|(A-\lambda)^{*} x_{n}\right\| \rightarrow 0$. Also, there exist $\mu, \mu^{\prime}$ in $\mathbb{C}$ such that both inequalities in (a) and (b) are satisfied. From the proof of (a) we see that $\mu$ can be chosen to be a cluster point of $\left\{\left(B x_{n}, x_{n}\right)\right\}$. From

$$
\begin{aligned}
\left|\left(B x_{n}, x_{n}\right)-\mu^{\prime}\right| & =\mid\left(x_{n},\left(B-\mu^{\prime}\right)^{*} x_{n} \mid\right. \\
& \leq\left\|\left(B-\mu^{\prime}\right)^{*} x_{n}\right\| \leq k\left\|(A-\lambda)^{*} x_{n}\right\|
\end{aligned}
$$


we see that $\mu=\mu^{\prime}$. This shows the existence of the function $f$ on $\sigma_{a p}(A) \cap \sigma_{a p}\left(A^{*}\right)^{*}$ such that both inequalities in (2) are satisfied. It remains to show that $f$ is a Lipschitz function.

Let $\lambda_{1}, \lambda_{2} \in \sigma_{a p}(A) \cap \sigma_{a p}\left(A^{*}\right)^{*}$. Then there exists a sequence $\left\{x_{n}\right\}$ of unit vectors such that $\left\|\left(A-\lambda_{2}\right) x_{n}\right\| \rightarrow 0$. Then, by (a), we also have $\left\|\left(B-f\left(\lambda_{2}\right)\right) x_{n}\right\| \rightarrow 0$. Hence

$$
\begin{aligned}
\left|f\left(\lambda_{1}\right)-f\left(\lambda_{2}\right)\right| & =\lim \left\|\left(B-f\left(\lambda_{2}\right)\right) x_{n}-\left(B-f\left(\lambda_{1}\right)\right) x_{n}\right\| \\
& =\lim \left\|\left(B-f\left(\lambda_{1}\right)\right) x_{n}\right\| \leq k \lim \inf \left\|\left(A-\lambda_{1}\right) x_{n}\right\| \\
& =k \liminf \left\|\left(A-\lambda_{1}\right) x_{n}-\left(A-\lambda_{2}\right) x_{n}\right\|=k\left|\lambda_{1}-\lambda_{2}\right| .
\end{aligned}
$$

\section{Main result.}

Theorem 4.1. Let $A \in \mathscr{B}(\mathscr{H})$ be a normal operator and $B \in \mathscr{B}(\mathscr{H})$. Then the following conditions are mutually equivalent.

(1) $D_{\mathrm{B}} \mathscr{C}_{2}(\mathscr{H}) \subseteq D_{\mathrm{A}} \mathscr{C}_{2}(\mathscr{H})$.

(2) $D_{B} \mathscr{C}_{1}(\mathscr{H}) \subseteq D_{A} \mathscr{B}(\mathscr{H})$.

(3) $B=f(A)$ for some Lipschitz function on $\sigma(A)$.

(4) $A B=B A$ and there exist $k>0$ and a Lipschitz function $f$ on $\sigma(A)$ such that, for all $\lambda \in \sigma(A)$, all $x \in \mathscr{H}$,

$$
\|(B-f(\lambda)) x\| \leq k\|(A-\lambda) x\| .
$$

(5) $B$ is normal and there exists a Lipschitz function fon $\sigma(A)$ such that $(B-f(\lambda)) \mathscr{H} \subseteq$ $(A-\lambda) \mathscr{H}$ for all $\lambda \in \sigma(A)$.

Proof. That (1) implies (2) is obvious. Next we assume (2) and prove (4) and (5). By means of Lemma 2.1, we can use an argument similar to that in the first part of the proof of Theorem 2.2 to show that there exists a positive constant $k>0$ such that, for each finite rank operator $X$, we have $\left\|D_{\mathrm{B}} X\right\| \leq k\left\|D_{\mathrm{A}} X\right\|_{1}$. Notice that, since $A$ is normal, $\sigma(A)=$ $\sigma_{a p}(A)=\sigma_{a p}\left(A^{*}\right)^{*}$. Hence, from Theorem 3.1, we see that there is a Lipschitz function $f$ on $\sigma(A)$ and a constant $k>0$ such that

$$
\begin{gathered}
\|(B-f(\lambda)) x\| \leq k\|(A-\lambda) x\|, \\
\left\|(B-f(A))^{*} x\right\| \leq k\left\|(A-\lambda)^{*} x\right\| .
\end{gathered}
$$

The last inequality guarantees the factorization $B-f(\lambda)=(A-\lambda) T$ for some $T \in \mathscr{B}(\mathscr{H})$ from which the inclusion $(B-f(\lambda)) \mathscr{H} \subseteq(A-\lambda) \mathscr{H}$ follows. To finish the proof of (4) and (5), it remains to show that $B$ is normal and commutes with $A$. For this purpose, it suffices to show that $B \in\{A\}^{\prime \prime}$. This follows from Corollary 2.3 and the fact that, for each $C$ in $\{A\}^{\prime},(B C-C B) \mathscr{C}_{1}(\mathscr{H}) \subseteq D_{A} \mathscr{B}(\mathscr{H})$, which can easily be deduced from the assumption $D_{\mathbf{B}} \mathscr{C}_{1}(\mathscr{H}) \subseteq D_{A} \mathscr{B}(\mathscr{H})$.

Now we assume (4) and proceed to show (3). Let $E(\cdot)$ be the spectral measure of $A$. Let $\varepsilon$ be an arbitrary positive number. Write $\sigma(A)$ as a disjoint union of finitely many disjoint Borel subsets $\sigma_{1}, \sigma_{2}, \ldots, \sigma_{n}$ of diameters less than $\varepsilon$ such that $f\left(\sigma_{1}\right), f\left(\sigma_{2}\right), \ldots, f\left(\sigma_{n}\right)$ also have diameters less than $\varepsilon$. For $x \in E\left(\sigma_{i}\right) \mathscr{H}$ and $\lambda_{j} \in \sigma_{i}$, we have

$$
\begin{aligned}
\|(B-f(A)) x\| & \leq\left\|\left(B-f\left(\lambda_{j}\right)\right) x\right\|+\left\|\left(f(A)-f\left(\lambda_{j}\right)\right) x\right\| \\
& \leq k\left\|\left(A-\lambda_{j}\right) x\right\|+\varepsilon\|x\| \leq(k+1) \varepsilon\|x\| .
\end{aligned}
$$


Since $B$ commutes with $A$, it commutes with $E\left(\sigma_{j}\right)$ for each $j$. Hence, for each $x \in \mathscr{H}$,

$$
\begin{aligned}
\|(B-f(A)) x\|^{2} & =\left\|(B-f(A)) \sum_{j=1}^{n} E\left(\sigma_{j}\right) x\right\|^{2} \\
& =\sum_{j=1}^{n}\left\|(B-f(A)) E\left(\sigma_{j}\right) x\right\|^{2} \\
& \leq(k+1)^{2} \varepsilon^{2} \sum_{j=1}^{n}\left\|E\left(\sigma_{j}\right) x\right\|^{2}=(k+1)^{2} \varepsilon^{2}\|x\|^{2} .
\end{aligned}
$$

Since $\varepsilon$ is arbitrary, we have $B=f(A)$.

Next we show that (5) implies (3). Let $E(\cdot)$ and $F(\cdot)$ be the spectral measure of $A$ and $B$ respectively. By Lemma 2.4, for a closed set $\tau$ in $\sigma(B)$, we have

$$
\begin{aligned}
F(\tau) \mathscr{H} & =\bigcap\{(B-\mu) \mathscr{H}: \mu \notin, \tau\} \\
& \subseteq \bigcap\left\{(B-f(\lambda)) \mathscr{H}: \lambda \notin f^{-1}(\tau)\right\} \\
& \subseteq \bigcap\left\{(A-\lambda) \mathscr{H}: \lambda \notin f^{-1}(\tau)\right\}=E\left(f^{-1}(\tau) \mathscr{H}\right) .
\end{aligned}
$$

On the other hand, there is an increasing sequence $\left\{\tau_{n}\right\}$ of closed sets in $\sigma(B)$ such that $\bigcup_{n=1}^{\infty} \tau_{n}=\sigma(B) \backslash \tau$ and hence

$$
\begin{aligned}
F(\sigma(B) \backslash \tau) \mathscr{H} & =\left\{\bigcup_{n} F\left(\tau_{n}\right) \mathscr{H}\right\}^{-} \\
& \subseteq\left\{\bigcup_{n} E\left(f^{-1}\left(\tau_{n}\right)\right) \mathscr{H}\right\}^{-}=E\left(\sigma(A) \backslash f^{-1}(\tau)\right) \mathscr{H} .
\end{aligned}
$$

Thus we conclude that $F(\tau)=E\left(f^{-1}(\tau)\right)$ for all closed sets $\tau$ in $\sigma(A)$. Now it is routine to show that $A=f(B)$.

Finally, we show that (3) implies (1). Again, let $E(\cdot), F(\cdot)$ be the spectral measures of $A$ and $B$ respectively. We assume that $f$ has the Lipschitz constant $k$. We write $\sigma(B)$ as the union of finitely many, mutually disjoint Borel subsets $\tau_{1}, \ldots, \tau_{n}$, each of which has diameter less than a given positive number $\varepsilon$. For $j=1, \ldots, n$, we write $f^{-1}\left(\tau_{j}\right)=$ $\bigcup\left\{\sigma_{i}: i \in I_{i}\right\}$, where $I_{1}, \ldots, I_{n}$ are mutually disjoint finite index sets and $\sigma_{i}$ are mutually disjoint Borel sets of diameter less than $\varepsilon$. We notice that $\sigma(A)=\bigcup_{i \in I} \sigma_{i}$, where $I=$ $I_{1} \cup I_{2} \cup \ldots \cup I_{n}$. For each $j$, take a point $\mu_{j}$ in $\tau_{j}$ and, for each $i$, take a point $\lambda_{i}$ in $\sigma_{i}$. We put

Then we have

$$
\begin{aligned}
& A_{\varepsilon}=\sum_{i \in I} \lambda_{i} E\left(\sigma_{i}\right) \\
& B_{\varepsilon}=\sum_{j=1}^{n} \mu_{j} F\left(\tau_{j}\right)=\sum_{i=1}^{n} \mu_{j} E\left(f^{-1}\left(\tau_{j}\right)\right) .
\end{aligned}
$$

$$
f\left(A_{\varepsilon}\right)=\sum_{i \in I} f\left(\lambda_{i}\right) E\left(\sigma_{i}\right)=\sum_{j=1}^{n} \sum_{i \in I_{i}} f\left(\lambda_{i}\right) E\left(\sigma_{i}\right) .
$$


Since

$$
\left\|\sum_{i \in I_{i}} f\left(\lambda_{i}\right) E\left(\sigma_{i}\right)-\mu_{j} E\left(f^{-1}\left(\tau_{j}\right)\right)\right\|<\varepsilon,
$$

we have $\left\|f\left(A_{\varepsilon}\right)-B_{\varepsilon}\right\|<\varepsilon$. Also notice that $\left\|A-A_{\varepsilon}\right\|<\varepsilon$ and $\left\|B-B_{\varepsilon}\right\|<\varepsilon$. Next, for $X \in \mathscr{C}_{2}(\mathscr{H})$, let $X_{\mathrm{rs}}=E\left(\sigma_{\mathrm{r}}\right) X E\left(\sigma_{s}\right)$ for $r, s \in I$. Then

$$
\begin{aligned}
\left\|D_{f\left(A_{e}\right)} X\right\|_{2}^{2} & =\left\|\sum_{r, s \in I}\left(f\left(\lambda_{r}\right)-f\left(\lambda_{s}\right)\right) X_{r s}\right\|_{2}^{2} \\
& =\sum_{r, s}\left|f\left(\lambda_{r}\right)-f\left(\lambda_{s}\right)\right|^{2}\left\|X_{r s}\right\|_{2}^{2} \\
& \leq k^{2} \sum_{r, s}\left|\lambda_{r}-\lambda_{s}\right|^{2}\left\|X_{r s}\right\|_{2}^{2}=k^{2}\left\|D_{A_{e}} X\right\|_{2}^{2} .
\end{aligned}
$$

Let $\varepsilon \rightarrow 0$, we have $\left\|D_{B} X\right\|_{2} \leq k\left\|D_{A} X\right\|_{2}$.

5. Remarks. In this final section we give some variants of Theorem 2.2 .

Proposition 5.1. For every $A$ in $\mathscr{B}(\mathscr{H}), D_{A} \mathscr{C}_{1}(\mathscr{H})$ does not contain any nonzero one-sided ideal.

Proof. Assume that $\mathscr{H} \otimes y_{0} \subseteq D_{\mathrm{A}} \mathscr{C}_{1}(\mathscr{H})$. Note that $\mathscr{C}_{1}(\mathscr{H})^{\prime}$ can be identified with $\mathscr{B}(\mathscr{H})$ and proceed as in the beginning of the proof of Theorem 2.2. We deduce that there exists a constant $k>0$ such that $\left\|X y_{0}\right\| \leq k\|A X-X A\|$ for all $X$ in $\mathscr{B}(\mathscr{H})$. Let $X=I$ in the above inequality; we obtain $y_{0}=0$.

For an operator $A$, it is natural to ask the following question: for each finite rank operator $F_{0}$ in $D_{A} \mathscr{B}(\mathscr{H})$, does there always exist a finite rank operator $F$ such that $F_{0}=D_{A} F$ ? Proposition 5.1 tells us that, if $A$ is an operator such that $D_{A} \mathscr{B}(\mathscr{H})$ contains a nonzero one-sided ideal, then the answer is no. In fact, if $\mathscr{H} \otimes y_{0} \subseteq D_{A} \mathscr{B}(\mathscr{H})$ with $y_{0} \neq 0$, then, by Proposition 5.1, there exists some $x \in \mathscr{H}$ such that $x \otimes y_{0} \notin D_{A} \mathscr{C}_{1}(\mathscr{H})$; but of course we have $x \otimes y_{0} \in D_{A} \mathscr{B}(\mathscr{H})$.

Proposition 5.2 (Weber [8, Theorem 3]). If $A$ is a Hilbert-Schmidt operator, then $D_{\mathrm{A}} \mathscr{B}(\mathscr{H})$ does not contain any nonzero one-sided ideal.

Proof. Assume that $\mathscr{H} \otimes y_{0} \subseteq D_{A} \mathscr{B}(\mathscr{H})$. We may regard $D_{\mathrm{A}}$ as a map from $\mathscr{B}(\mathscr{H})$ into $\mathscr{C}_{2}(\mathscr{H})$ and hence its transpose $D_{A}^{\prime}$ becomes a map from $\mathscr{C}_{2}(\mathscr{H})$ into $\mathscr{C}_{1}(\mathscr{H})$. Hence there exists $k>0$ such that $\left\|X y_{0}\right\| \leq k\|A X-X A\|_{1}$ for all $X \in \mathscr{C}_{2}(\mathscr{H})$. Letting $X=A$, we obtain $A y_{0}=0$. Now the proposition follows from the following lemma which is implicit in [8].

LEMMA 5.3. If $A$ is an operator in $\mathscr{B}(\mathscr{H})$ with the property that, for each $y_{0} \in \mathscr{H}$, $\mathscr{H} \otimes y_{0} \subseteq D_{A} \mathscr{B}(\mathscr{H})$ implies $A y_{0}=0$, then $D_{A} \mathscr{B}(\mathscr{H})$ does not have any nonzero right ideal.

Proof. Suppose that $\mathscr{H} \otimes y_{0} \subseteq D_{A} \mathscr{B}(\mathscr{H})$. We have to show that $y_{0}=0$. By the assumed property of $A$, we have $A y_{0}=0$. On the other hand, there exists $X \in \mathscr{B}(\mathscr{H})$ such that $y_{0} \otimes y_{0}=A X-X A$. Hence $\left\|y_{0}\right\|^{2} y_{0}=\left(y_{0} \otimes y_{0}\right) y_{0}=A X y_{0}$. It suffices to show that $\mathscr{H} \otimes X y_{0} \subseteq D_{A} \mathscr{B}(\mathscr{H})$ since, in view of the assumed property of $A$, this inclusion implies 
$A X y_{0}=0$, which in turn implies $y_{0}=0$. Now for each $y \in \mathscr{H}$, there exists $Y \in \mathscr{B}(\mathscr{H})$ such that $y \otimes y_{0}=A Y-Y A$ and hence

$$
\begin{aligned}
y \otimes X y_{0} & =X\left(y \otimes y_{0}\right)=X A Y-X Y A \\
& =(X A-A X) Y+D_{A}(X Y)=Y^{*} y_{0} \otimes y_{0}+D_{A}(X Y) \in D_{A} \mathscr{B}(\mathscr{C}) .
\end{aligned}
$$

\section{REFERENCES}

1. K. F. Clancy, On the local spectra of seminormal operators, Proc. Amer. Math. Soc. 72 (1978), 473-479.

2. M. R. Embry, Factorizations of operators on Banach space, Proc. Amer. Math. Soc. 38 (1973), 587-590.

3. B. E. Johnson, Continuity of linear operators commuting with continuous linear operators, Trans. Amer. Math. Soc. 128 (1967), 88-102.

4. B. E. Johnson and J. P. Williams, The range of a normal derivation, Pacific J. Math. 58 (1975), 105-122. 33-36.

5. C. R. Putnam, Ranges of normal and subnormal operators, Michigan Math. J. 18 (1971),

6. R. Schatten, Norm ideals of completely continuous operators (Springer-Verlag, 1960).

7. J. G. Stampfli, Derivations on $\mathscr{B}(\mathscr{H})$ : the range, Illinois J. Math. 17 (1973), 518-524.

8. R. E. Weber, The range of a derivation and ideals, Pacific J. Math. 50 (1974), 617-624.

9. J. P. Williams, On the range of a derivation II, Proc. Roy. Irish Acad. Sect. A 74 (1974), 299-310.

Department of Mathematics

UNIVERSITY OF TORONTO

TORONTO, CANADA

M5S 1A1 